\title{
Comme un voleur qui enrichirait la maison cambriolée : la bibliothèque d'André Schwarz-Bart
}

Jean-Pierre Orban

\section{(2) OpenEdition}

1 Journals

Édition électronique

URL : https://journals.openedition.org/genesis/5703

DOI : 10.4000/genesis.5703

ISSN : 2268-1590

Éditeur :

Presses universitaires de Paris Sorbonne (PUPS), Société internationale de génétique artistique littéraire et scientifique (SIGALES)

Édition imprimée

Date de publication : 15 décembre 2020

Pagination : 111-126

ISBN : 979-10-231-0704-3

ISSN : 1167-5101

\section{Référence électronique}

Jean-Pierre Orban, « Comme un voleur qui enrichirait la maison cambriolée : la bibliothèque d'André Schwarz-Bart », Genesis [En ligne], 51 | 2020, mis en ligne le 20 décembre 2021, consulté le 07 février 2022. URL : http://journals.openedition.org/genesis/5703; DOI : https://doi.org/10.4000/genesis. 5703

Ce document a été généré automatiquement le 7 février 2022

Tous droits réservés 


\title{
Comme un voleur qui enrichirait la maison cambriolée : la bibliothèque d'André Schwarz-Bart
}

\author{
Jean-Pierre Orban
}

\begin{abstract}
Comme David, Haïm assembla les ouvrages de sa bibliothèque sur la Shoah, les étoiles, l'histoire de la terre, l'histoire des hommes depuis l'Afrique, l'histoire de toutes les violences commises depuis le début des Temps, l'histoire juive, l'histoire sur l'histoire des juifs, les poètes et écrivains qu'il aimait et puis il s'en alla'.
\end{abstract}

1 En novembre 1959, André Schwarz-Bart remporte le prix Goncourt avec un premier roman, Le Dernier des Justes (aux Éditions du Seuil), qui retrace six siècles d'antisémitisme ayant mené à la Shoah. Succès public considérable, le livre d'un inconnu du monde littéraire est reçu avec des réserves par une partie du milieu intellectuel juif, soutenue par la revue Arts sous la direction d'André Parinaud ${ }^{2}$. Les uns ou les autres l'accusent d'erreurs historiques et de plagiats, et lui reprochent sa vision trop christique, à leurs yeux, du Juif ${ }^{3}$. Meurtri, André Schwarz-Bart quitte la France pour la Guyane, puis s'installe au Sénégal et en Guadeloupe avec celle qui, entre-temps, est devenue son épouse, la Guadeloupéenne Simone Brumant.

2 André Schwarz-Bart se consacre alors à l'écriture d'un cycle-conçu avant la publication du Dernier des Justes, avant aussi la rencontre de Simone Brumant - consacré à l'histoire de l'esclavage de l'Afrique aux Antilles, drame en miroir de la Shoah, à porter lui aussi au compte de l'Occident. En 1967, toujours aux Éditions du Seuil, il publie Un plat de porc aux bananes vertes, cosigné avec son épouse qui a pris le nom de Simone Schwarz-Bart. Et en 1972, il fait paraître sous son nom seul La Mulâtresse Solitude (Seuil), histoire romancée d'une Négresse marronne, héroïne réelle de la révolte contre le rétablissement de l'esclavage sous Bonaparte. L'histoire ainsi racontée par Schwarz- 
Bart deviendra plus tard un mythe en Guadeloupe, mais la publication est accueillie froidement par les milieux intellectuels antillais à une époque où commencent à s'affirmer les mouvements indépendantistes et créolisants, et, au début, avec une certaine indifférence par le public général. La légitimité de l'auteur blanc, métropolitain et juif pour parler de la tragédie noire se voit parfois mise en question ${ }^{4}$.

S'interrogeant de plus en plus sur la voie qu'il a prise littérairement et humainement, marqué par l'accueil réservé des deux " peuples » dont il voulait décrire les drames en miroir ${ }^{5}$ et déçu par l'accueil critique de ses romans «antillais $»^{6}$, André Schwarz-Bart cesse de publier ${ }^{7}$ en son nom propre. Il entre « en silence " public jusqu'à sa mort en 2006. Il ne cesse pourtant d'écrire. S'il n'achève pas le «cycle antillais " (laissant des pans entiers de brouillons qui seront rassemblés dans des publications posthumes), ni ne semble avoir écrit le livre qui aurait dû réunir les deux fils, juif et noir, de son œuvre (" En souvenir du xx siècle »), il se remet, surtout à partir du milieu des années quatrevingt, à une nouvelle œuvre juive. Cette œuvre qu'il projette, selon ses notes, sous différentes formes successives, doit parler de la situation des rescapés de la Shoah. En partie autobiographique, le projet dénommé la plupart du temps Kaddish (K.) met en scène un double, Haïm, parfois en dialogue avec André Schwarz-Bart (AS, ASB).

Fig. 1 : Plan « en étoile » du projet de « roman juif », Kaddish

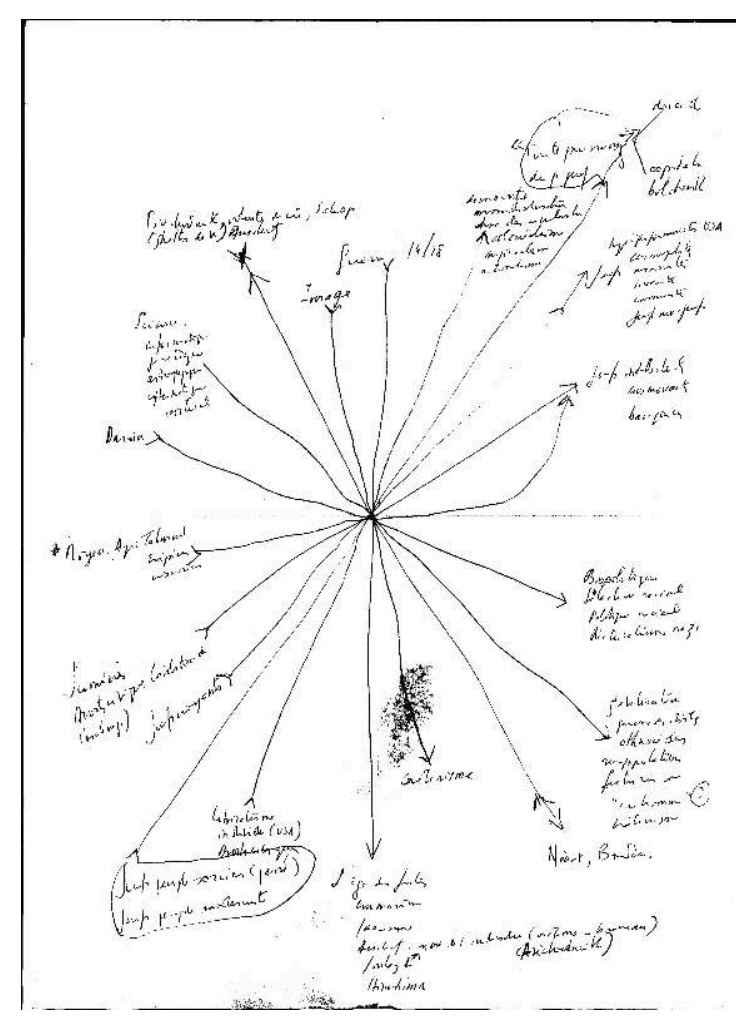

Feuillet libre (format A4) retrouvé par Francine Kaufmann lors de son classement des papiers et de la bibliothèque d'André Schwarz-Bart à Goyave, Guadeloupe, après la mort de l'écrivain.

(c) Archives personnelles de Simone Schwarz-Bart

Rongé par le doute et par la question de la légitimité à écrire, lui survivant, sur les morts, paralysé par un éventuel accueil négatif du public juif ${ }^{8}$, poussé par des penchants dépressifs, André Schwarz-Bart fait disparaître nombre de ses documents manuscrits/tapuscrits, sans que l'on sache précisément dans quelle proportion'. Des 
anecdotes circulent selon lesquelles il porte lui-même des sacs de documents à la déchetterie de peur que ses proches ne les récupèrent dans ses poubelles. Que ces récits soient vrais ou mythifiés, l'inhibition n'en est pas moins indubitable : elle s'exprime dans sa correspondance (notamment avec ses éditeurs ${ }^{10}$ ) et plusieurs notes dans les carnets ou les feuillets libres qui ont survécu à la destruction. Le mot " naufrage » est central dans la dernière partie de sa vie. Ainsi dans cet extrait de L'Étoile du matin (ÉdM), roman posthume constitué lui-même de pièces sauvées, où le mot "naufrage " semble désigner à la fois l'engloutissement et les débris surnageant, comme si ces derniers constituaient le désastre lui-même : « Mais il savait maintenant qu'il n'écrirait pas ce livre: peut-être réunirait-il quelques pages pour ses amis, pages qui constitueraient le naufrage d'un livre, ce qui correspondait mieux au naufrage de son esprit depuis Auschwitz ${ }^{11}$.»

Qu'est-il resté de ce naufrage en termes d'archives, hors correspondance, photographies de voyage, dessins de l'auteur ou dossiers de réception et d'adaptations, et traductions des œuvres? Peu de manuscrits ou tapuscrits originaux des romans publiés, l'une ou l'autre version antérieure du Dernier des Justes, beaucoup de fragments et bouts d'ébauches des romans du cycle antillais inachevée ${ }^{12}$, une bonne vingtaine de cahiers, carnets ou bloc-notes, ainsi que des feuillets libres de tous types rassemblant des réflexions générales, personnelles ou intimes, et des notes sur les œuvres en cours ou en projet, ainsi qu'un certain nombre de cassettes qui font office de carnets de notes audio.

6 Enfin, pièce maîtresse de ces archives, une bibliothèque dont on peut penser qu'elle a échappé au naufrage en raison de sa nature même: si on s'autorise à jeter ses propres brouillons ou ébauches, on ne se permet pas, ou difficilement, de détruire les œuvres de ses pairs ${ }^{13}$. Et comme André Schwarz-Bart écrivait sur tout type de support et en particulier dans les livres de sa bibliothèque, celle-ci représente, en quelque sorte, une embarcation de salut dans son propre naufrage. Un assemblage de livres sur lequel, pendant les vingt ou vingt-cinq dernières années de sa vie, il aura vécu solitaire tout en étant en lien avec le monde. Un radeau-bibliothèque où il aura pensé et écrit en un colloque singulier avec les autres. C'est ce rapport complexe aux livres des autres qu'il convient sinon d'élucider, au moins de décrire.

\section{Bibliothèque réceptacle et creuset d'une œuvre inachevée}

7 Pour préservée qu'elle ait été dans l'errance et le naufrage schwarz-bartien, cette bibliothèque n'a cependant pas traversé indemne le temps. Elle a subi les déménagements successifs, en Europe (Paris, Lausanne, Évian) et entre les continents, avec chaque fois des pertes ${ }^{14}$ relatées par Simone Schwarz-Bart et qui auraient également, parfois, concerné les documents manuscrits ${ }^{15}$. En 1978, cependant, le couple s'installe définitivement à Goyave en Guadeloupe: dans une maison surnommée $L a$ Souvenance, un étage est réservé à André, dont le centre, entouré d'une coursive, est occupé par une chambre-bureau dont les murs tapissés de livres entourent le lit et la table de travail. C'est dans cet espace qu'André vit la plupart du temps seul, passant de sa bibliothèque à son petit salon et à son immense baignoire où il passe de longues heures à lire jusqu'à s'endormir : les livres maculés de taches d'encre diluées dans l'eau en témoignent (fig. 2). 
Fig. 2 : Métonymie du naufrage

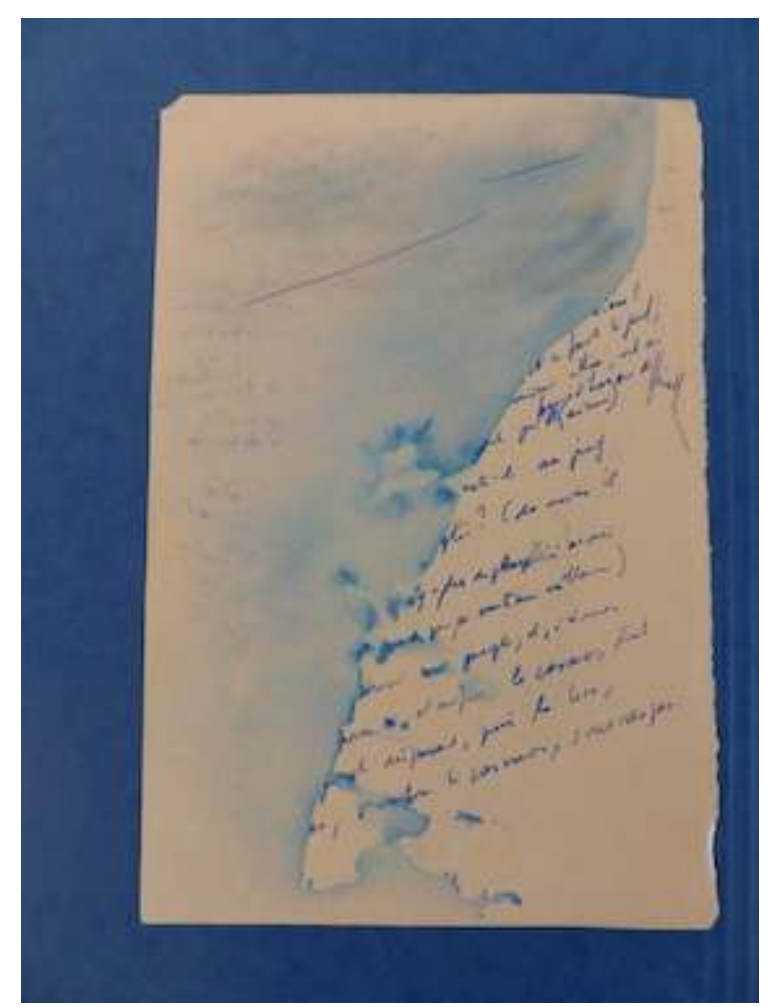

Page arrachée d'un livre (sans doute Marcel Conche, Vivre et philosopher. Réponses aux questions de Lucie Laveggi, Paris, PUF, 1992) et tachée d'encre diluée.

(C) BnF, Fonds Schwarz-Bart

Après sa mort, le lieu est resté plusieurs années délaissé, les ouvrages rangés dans des cartons pour les protéger des menaces de cyclone et des fuites dans le toit. Jusqu'à ce qu'à la fin des années 2000 et au début des années 2010, la chercheuse Francine Kaufmann explore, dépouille et classe les documents personnels d'André Schwarz-Bart à Goyave. Lors de ce classement, elle réorganise la bibliothèque selon un ordre qu'elle suppose être celui de l'écrivain. En 2018, dans le cadre du don progressif des archives Schwarz-Bart à la Bibliothèque nationale de France, $80 \%$ de cette bibliothèque, à savoir quelque 1300 titres comportant des interventions d'André Schwarz-Bart, ont été transférés à Paris, fait de plus en plus rare à la BnF par manque de place et en raison des coûts de transfert, de traitement et de gestion. Après une année de quarantaine et de traitement de décontamination, les ouvrages sont accessibles à la recherche, depuis octobre 2019.

9 Il est difficile de déterminer dans quelle mesure cette bibliothèque telle qu'elle nous est parvenue reflète les sources de l'œuvre publiée de(s) Schwarz-Bart.

On sait qu'André Schwarz-Bart a beaucoup fréquenté les bibliothèques publiques et universitaires (la bibliothèque Sainte-Geneviève, la BnF à Paris) au cours de la rédaction du Dernier des Justes et de la préparation du cycle antillais. Parmi les exemplaires de titres présents dans le dernier état de sa bibliothèque ${ }^{16}$, peu semblent avoir directement servi à alimenter son premier roman. Plusieurs items (titres et numéros de revues) datent de manière claire ou vraisemblable d'avant le retour définitif en Guadeloupe en 1978, ainsi Plus profond que l'abîme de Manès Sperber (Calmann Levy, 
1950) ou la revue Targoum (Paris) qui n'a existé que de 1954 à 1956 : des marques de stylo ou crayon rouge, moins habituelles ultérieurement, permettent de le supposer. Mais les annotations et autres interventions ne paraissent pas souvent liées au Dernier des Justes. Nombre d'ouvrages ont dû être achetés et consultés plusieurs fois ${ }^{17}$ et les annotations au stylo et crayon rouge, bien qu'anciennes, datent peut-être des années postérieures à la publication du roman fondateur. Il est également possible que l'habitude d'annoter les ouvrages parfois jusqu'à la surcharge soit apparue assez tard dans la vie de Schwarz-Bart. On dispose donc de peu de traces permettant de déterminer un dispositif exogénétique relatif au Dernier des Justes.

11 À l'inverse, en aval de la publication, dans un titre de 1992, Histoire des Juifs de Pologne, $d u X I^{e}$ siècle à nos jours (Paris, PUF), qui couvre chronologiquement une bonne partie du sujet du Dernier des Justes, on trouve plusieurs marques «DdJ » en marge de passages qui, plus de trente ans plus tard, renvoient Schwarz-Bart à son premier roman et aux polémiques qui ont entouré sa parution. Une phrase soulignée, «Dans l'esprit de justification du bien par la souffrance»(p. 81), évoque ainsi le débat sur l'attitude christique des personnages du Dernier des Justes. Ici, des annotations n'indiquent pas une genèse, mais nous renseignent sur un processus de réception par l'auteur luimême, une relecture permanente de sa propre œuvre, dont on retrouve un autre exemple en marge d'un article de François Bédarida, "La mémoire contre l'histoire ", dans un numéro de la revue Esprit de juillet 1993 sur « Le poids de la mémoire » (p. 9) :

En fait, les difficultés de ma tentative tenaient au fait que ma démarche voulait à la fois traiter de la mémoire et de l'Histoire. S'ajoutant au crime de la fiction, c'était insoutenable.

De la même façon que pour Le Dernier des Justes - alors qu'on sait, par les témoignages de ses proches et par la boulimie de lectures dont il fait preuve dans ses carnets et ses notes, que Schwarz-Bart effectuait des recherches nombreuses et méticuleuses lors de la préparation de ses romans -, les sources livresques du cycle antillais sont, à de rares exceptions, absentes de sa bibliothèque à Goyave telle qu'elle nous est parvenue ${ }^{18}$. On ne les retrouve pas plus dans un carnet où il a noté presqu'entièrement, selon une numérotation qui n'a pas encore révélé sa logique, l'inventaire de cette bibliothèque (fig. 3) au temps où il l'a constituée.

Fig. 3 : Photo de deux pages du carnet-inventaire de sa bibliothèque, par André Schwarz-Bart luimême

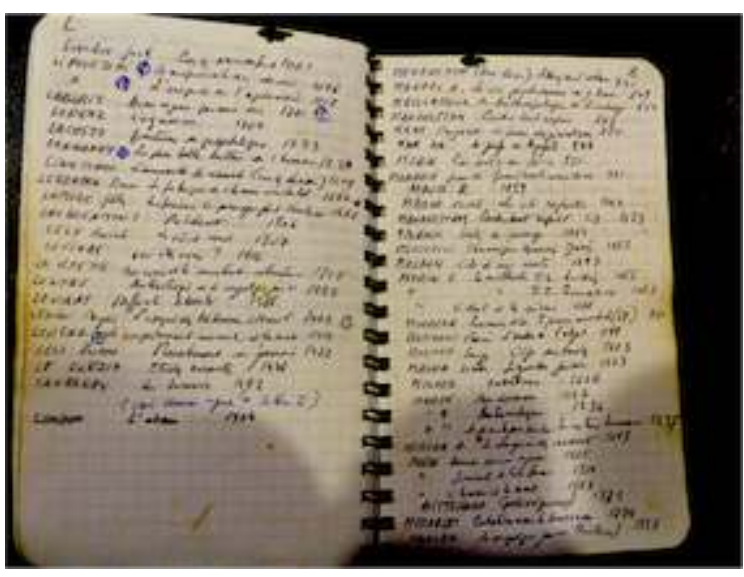

(C) BnF, Fonds Schwarz-Bart 
En réalité, pour l'essentiel, la bibliothèque de Goyave est liée, par son contenu et ses annotations, à ce qui constituait le retour d'André Schwarz-Bart au thème juif, la suite ou comme l'écho sismique du Dernier des Justes et du génocide juif lui-même, après son " détour " par le thème de l'esclavage noir. Durant plus de vingt ans, cette bibliothèque, qui comporte aussi de nombreux journaux et magazines aux textes et annonces entrelardés de notes manuscrites, sera ainsi le réceptacle de ses réflexions sur ce thème et le creuset d'une œuvre qui, en grande partie, n'ira pas jusqu'au bout de son écriture.

Fig. 4 : Publicité de France Telecom dans Le Monde entrelardée d'une note manuscrite d'André Schwarz-Bart sur Haïm, personnage de "Kaddish »

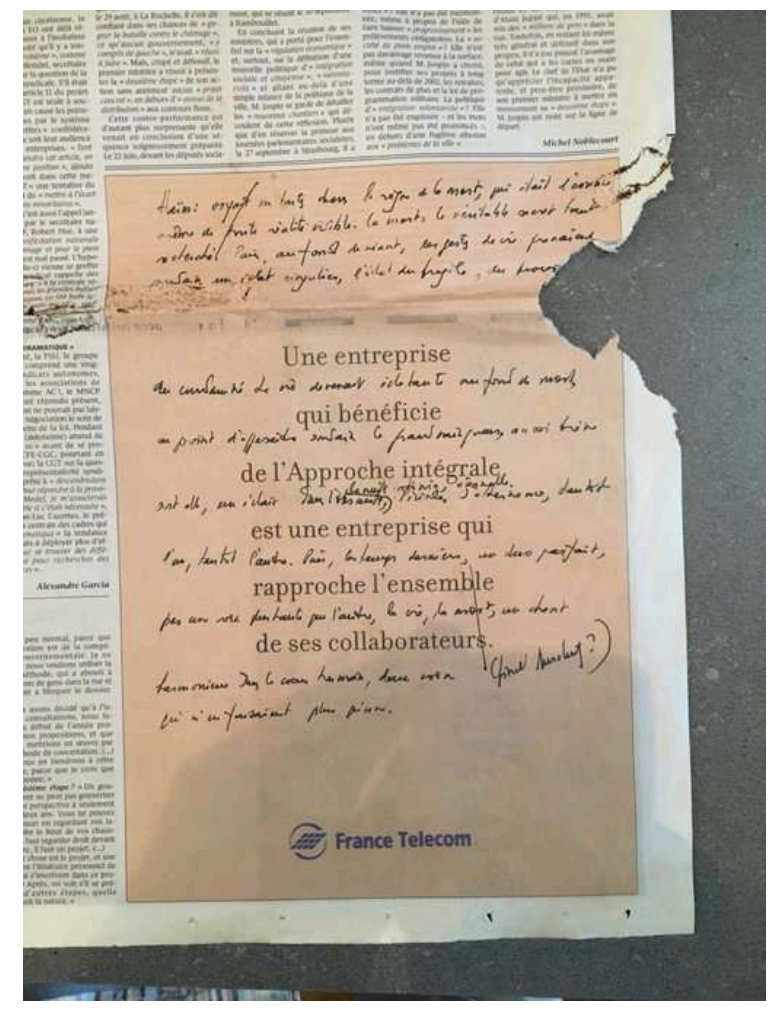

(c) BnF, Fonds Schwarz-Bart

\section{Un rapport de violence et de réappropriation avec les textes d'autrui}

Si on reprend la distinction établie par Daniel Ferrer dans Bibliothèques d'écrivains ${ }^{19}$ entre « extracteurs » et " marginalistes », André Schwarz-Bart fait résolument partie de ces derniers. En poussant à l'extrême leur comportement. C'est qu'à la manière d'un Stendhal, il ne se contente pas des marges des textes: ce sont tous les espaces blancs qu'il envahit de commentaires et notes de travail sur son projet "Kaddish », mais aussi de notes prosaïques (numéros de téléphone, rendez-vous, rappel d'une tâche à faire ou prix d'un objet). Davantage encore, l'attitude iconoclaste à l'égard des ouvrages va jusqu'à très fréquemment en maltraiter les pages en les cornant, les recornant et les pliant, en les couvrant de signes dont la recherche devra établir la signification et la hiérarchie, en griffonnant, surtout dans les dernières pages - ainsi dans les titres de la collection -, des dessins géométriques qui les recouvrent jusqu'à en cacher le contenu. 
Mais il va plus loin. Sans aucun respect pour l'intégrité matérielle des ouvrages, il découpe ou arrache les pages sur lesquelles il a annoté ses réflexions personnelles ou notes d'écriture. La plupart du temps, il s'agit des pages de garde ou des derniers feuillets du livre, mais cela va souvent jusqu'aux premières pages du texte. Ces pages, il les met de côté comme des feuillets de blocs-notes. Il arrive que la découpe, tout en demeurant radicale, soit plus précise, précautionneuse, comme chirurgicale : ainsi dans l'Encyclopedia Judaïca qu'il ne se permet pas cette fois, dirait-on, de surcharger de sa propre écriture, mais dont il enlève au cutter de nombreuses pages liées à ses recherches ou à ses intérêts, les replaçant ensuite à l'endroit où il les a subtilisées, comme un voleur discret ou contrit qui, pour le faire oublier ou pardonner, remettrait en place le produit de son vol après en avoir tiré le profit désiré.

Fig. 5 : Pages découpées ou arrachées, classées et rangées

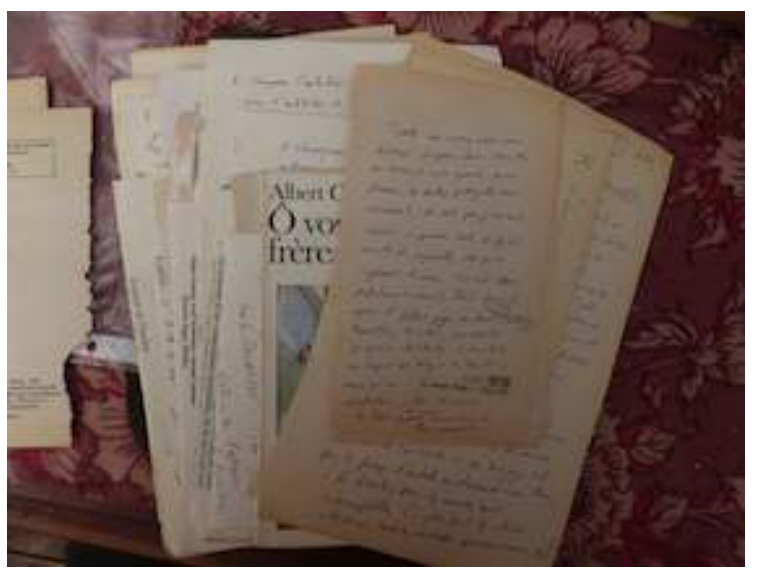

(c) BnF, Fonds Schwarz-Bart

Le rapport aux livres des autres témoigne donc d'une certaine violence, qu'elle soit froide ou pas, rachetée ou non. À quelques exceptions près (tels les ouvrages d'art), l'objet-livre, au moins, ne semble détenir pour lui aucune valeur. Mais le contenu luimême est chahuté, charcuté, pris à partie. Schwarz-Bart est né dans un milieu où le livre, hormis religieux, était peu présent, sinon absent. «Les livres, la musique appartenaient à mes yeux à un univers dont je ne faisais pas partie ", dit-il dans un entretien de $1959^{20}$.

La guerre et la déportation de ses parents et de ses frères l'ont conduit à interrompre tôt ses études secondaires, avant qu'il ne les reprenne plus tard. Entre sa pratique de la langue parlée, yiddish à la maison, français populaire dans la rue et ce qu'il appelait la "langue de la bourgeoisie ${ }^{21}$ ", il y a un "fossé » qu'il comblera au fil des ans par une lecture vorace et approfondie, mais demeurera une lésion aigüe empreinte d'un désir de revanche existentiel mais aussi politique ${ }^{22}$.

On peut donc parler, chez Schwarz-Bart, d'une "expropriation»- ici, sociale et politique - des textes d'autrui comme le fait Daniel Ferrer à propos de Stendhal ${ }^{23}$, mais il convient en même temps d'évoquer un processus de "réappropriation", car les textes sont ingurgités, assaisonnés d'une masse d'encre et de commentaires, assimilés jusqu'à les faire siens ou à les régurgiter comme autres, différents, voire adversaires ou ennemis: dans un exemplaire de L'Univers concentrationnaire, de David Rousset ${ }^{24}$, ouvrage fondateur de la réflexion sur le thème concentrationnaire au centre de la 
pensée et de l'œuvre d'André Schwarz-Bart, celui-ci tour à tour souligne des passages destinés à alimenter son projet "Kaddish" - ainsi "gestes hallucinés et raides de mécaniques enrayées » (p. 23, passage marqué de la lettre «K. ») -, conteste, corrige, se moque («ronflant: on se croirait dans un*e* *romance* littéraire des années 30 », p. 71) ou injurie: «le communiste avant le juif, naturellement, vieille crapule néostalinienne!» (p. 120).

Tout au long de ses lectures, André Schwarz-Bart absorbe, mange, dévore donc et, en marge, à côté, contre, par-dessus les textes de ceux qui deviennent ses pairs, pose et dépose ses propres écritures à la manière du coucou, «du parasite qui implante ses petits dans le nid, voire dans la chair de l'animal hôte ${ }^{25} »$ ou plus encore, en une occupation du territoire d'autrui, allié, concurrent ou adversaire. Le phénomène intertextuel est, dès lors, certes celui d'une interaction, mais une interaction qui opère peu dans un esprit de complicité, et assurément pas dans un entre-soi, plutôt à l'inverse en un processus, sinon une stratégie de confrontation parfois dure, front contre front, marge contre centre de la page, encre contre encre.

\section{De l'« arobase » à l'invective}

On a de la sorte un éventail d'interventions qui constituent autant de nuances de relation, de degrés de confrontation et de types de juxtaposition.

Sans nullement en épuiser l'inventaire, on relèvera les cornements et recornements de pages, les soulignements simples, doubles, couvrant parfois toute la page, les passages ou pages entièrement barrés, les marques marginales aux formes diverses et significations multiples - traits verticaux, crochets, croix, cercles, carrés, carrés avec croix intérieure, volutes, points noirs, points creux, points d'interrogation bien sûr et même un signe similaire à une arobase (@) - ceci dans différentes couleurs indiquant sans doute des périodes d'annotation différentes.

Viennent ensuite les commentaires, questions («Quelle est la part de la littérature [...] et ce qui échappe à la littérature? » dans L'Espèce humaine de Robert Antelme $\left.{ }^{26}\right)$, les appréciations ( TB » ou " Débile» dans Les Ombres errantes de Pascal Quignard ${ }^{27}$ ) qui peuvent précéder des moqueries, des prises à parties ou des apostrophes (dans le même ouvrage de Quignard: "Qu'en sais-tu? As-tu demandé ?»), des ricanements (« Ha! ha! » dans Les Battements du monde: Dialogue d'Alain Finkielkraut et Peter Sloterdijk ${ }^{28}$ ) ou des invectives ( Connasse » dans un article de Viviane Forrester ${ }^{29}$ ).

Parfois, Schwarz-Bart confronte également deux auteurs dans l'ouvrage de l'un des deux, ainsi Antelme et Rousset dans le livre de ce dernier: "Ces notes impressionnantes ne nous donnent pas la loi des camps : peut-être sont-elles destinées à masquer pas mal de choses. C'est aussi la "technique" de Robert Antelme ${ }^{30}$.» 
Fig. 6 : «Vieille crapule néostalinienne !»

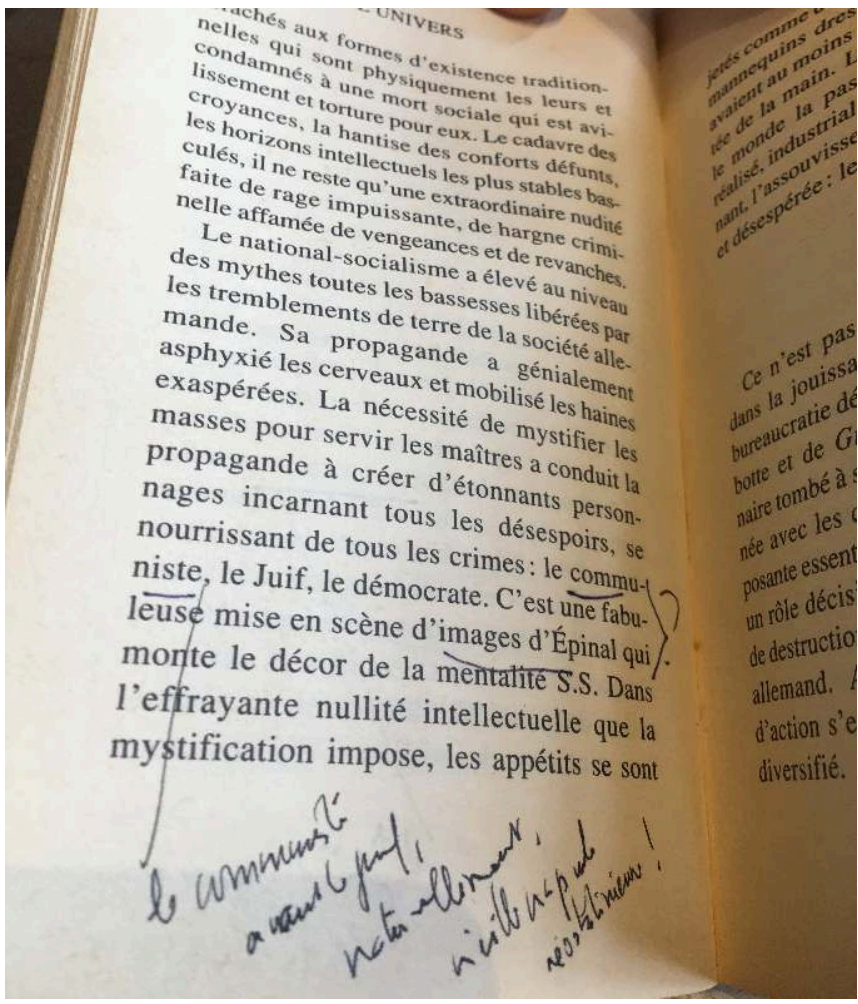

André Schwarz-Bart invective David Rousset dans L'Univers concentrationnaire (édition de poche, 1993)

(c) BnF, Fonds Schwarz-Bart

\section{Vers L'Étoile du matin en passant par Bouddha et Lady Macbeth}

Génétiquement parlant, l'importance de la bibliothèque d'André Schwarz-Bart ne se trouve pas, cependant, dans ses notes de lecture, même si ces lectures, on l'imagine, irriguaient sa pensée et, dès lors, son œuvre et ses projets littéraires. L'intérêt se trouve bien davantage dans « l'espace transactionnel ${ }^{31}{ }^{1}$ " que la bibliothèque constitue et où se développe une dynamique intime et, pour une grande part, mystérieuse entre livres et manuscrits, un espace " où l'écriture en train de se faire s'articule sur le déjà-écrit ${ }^{32}$ ». Un processus où se mêlent donc exogenèse et endogenèse, où se brouille la frontière entre les deux. Un passage de l'une à l'autre en un processus d'absorption de la première dans l'autre, tel que le décrit Pierre-Marc de Biasi en relativisant l'opposition des deux notions ${ }^{33}$, mais ici avec un effet direct et dans une proximité immédiate, une contiguïté maximale entre le « lire » et l'« écrire ».

Au milieu des années quatre-vingt, on l'a dit, André Schwarz-Bart entame en effet le projet « Kaddish » comme il le nomme lui-même la plupart du temps : «Titre provisoire du livre : Kaddish. C'est en effet un des thèmes fondamentaux : l'histoire d'un Kaddish impossible et qui pourtant devient possible à la fin, au moins je le crois, je l'espère ", écrit-il en décembre 1985 à Francine Kaufmann en lui répétant en juin de la même année : «J'ai entrepris il y a six mois une sorte de "conte fantastique" qui commence en Pologne au début du siècle dernier et s'achève de nos jours à Jérusalem. J'espère le 
terminer un jour ${ }^{34}$.»Ce projet s'orientera vers deux formes différentes : théâtrale et, assez vite, romanesque.

À la toute fin de sa vie, comme face à une urgence qu'il ressent, l'auteur dictera à son épouse et ses fils des blocs entiers de cette œuvre en chantier. On possède également un tapuscrit de ces chapitres. Après la mort d'André en 2006, avec l'aide notamment de Francine Kaufmann, Simone retrouvera d'autres fragments et des ébauches partielles de la même œuvre. Elle reliera les blocs cohérents aux fragments, comblera les lacunes, aménagera les liaisons, modifiera le texte et procédera à des coupes quand elle le jugera nécessaire. Il en résultera un roman auquel elle donnera le titre lancé quelques jours par André avant sa mort : L'Étoile du matin ${ }^{35}$.

Ce roman s'est élaboré en grande partie au fil des ans (entre 1985 et 2006) à partir de notes déposées en marge des ouvrages de sa bibliothèque et dans des carnets qui en constituent le relais. On retrouve dans ces notes les thèmes du roman posthume: le destin du peuple juif, la déportation et le génocide, les rescapés et la vie "après ». Certains lieux: Varsovie, Paris, Auschwitz. Plusieurs personnages: Haïm, le double d'André Schwarz-Bart, à qui L'Étoile du matin donne un passé, André Schwarz-Bart luimême qui d'interlocuteur d'Haïm dans les notes devient une référence dans le roman publié avec de fausses autocitations ou des vestiges de ses interventions manuscrites placées en exergue de chapitres, le pédagogue Janusz Korczak fondateur d'un orphelinat dans le ghetto de Varsovie, ou encore le ou la «chroniqueur» ou " chroniqueuse » dont on peut penser que Linemarie ${ }^{36}$, l'immortelle qui découvre les débris ou « traces poussiéreuses » (ÉdM, p. 23) du livre-naufrage, ou le «narrateur » qui apparaît dans une « Note pour le Livre imaginaire » (ÉdM, p. 205) sont des avatars.

Entre le livre d'autrui et la note manuscrite, marginale, d'André Schwarz-Bart, l'interaction est complexe et souvent difficile à déterminer. L'écriture est-elle déclenchée par le contenu du livre, directement ou par des voies détournées, sinon inconscientes, d'association? Ou le rapport est-il inexistant, les pages des autres ne servant que comme papier libre ? Tour à tour ou simultanément les trois.

Car si le sujet du livre lu est souvent lié à l'œuvre en gestation et semble offrir un monde-cadre à l'imagination (c'est le cas des titres sur les camps, la barbarie moderne ou l'histoire du judaïsme, des œuvres d'Arnold Mandel à celles de Vassili Grossman), ce lien est loin de toujours se vérifier. On trouve ainsi des notes importantes intégrées à des publicités parfaitement prosaïques pour Gucci ou une marque de voiture dans des journaux et magazines généralistes tels que Libération ou Le Nouvel Observateur. De la même manière, une idée - «K. Goyave ? La femme lui prépare un repas juif (version [illis.] de Varsovie) » - est notée en haut de l'introduction de L'Enseignement du Bouddha de Walpala Rahula (Seuil, coll. « Points », 1995) et une note sur le personnage capital de la mère d'André Schwarz-Bart est nichée dans un roman policier de Mary Higgins Clark (Le Fantôme de Lady Macbeth, Albin Michel, 1989).

30 Autre exemple d'écart apparent entre l'annotation et le contenu du livre où elle est déposée: dans une édition de L'Homme et la Ville d'Henri Laborit ${ }^{37}$, on découvre plusieurs notes qui, mêlées ou redistribuées, ébauchent divers épisodes finaux de L'Étoile du matin. Ainsi la liaison entre les personnages d'Haïm et de Sarah :

Immobiles, puis Haïm s'approche d'elle, il tremble, elle tremble aussi, il défait en tremblant la robe, elle défait en tremblant la chemise d'Haïm, il [illis.] la culotte, elle baisse le pantalon et puis le slip de l'homme, *immobile* et tremblante, puis éclatent de rire et vont *se serrer* l'un contre l'autre, tombant ensemble dans le lit, 
riant.

Le temps passe. Ils sont heureux, mais il a toujours des absences [...]. Elle se réveille le voit dans le lit «absent» du monde [...] il est à Auschwitz. Puis un jour lui annonce qu'elle est enceinte. Etc. Etc. Plus d'absence. La fin Voyage Plus d'absence. (Note consignée dans les premières pages de L'Homme et la Ville d'Henri Laborit. Voir fig. 7)

Ils se retrouvèrent en tremblant à Jérusalem. Tous deux demeurèrent longtemps au bord du lit d'hôtel, échangeant des regards étonnés, effrayés. Puis ils éclatèrent de rire et tout fut bien. (ÉdM, p. 221)

Un matin, Sarah lui murmura à l'oreille qu'elle était enceinte. (ÉdM, p. 230)

Ça ne peut plus durer, il faut que tu m'emmènes en Pologne. (ÉdM, p. 233)

[Chapitre IV « Le voyage à Auschwitz »] (ÉdM, p. 237)

Cette nuit-là, il fit un rêve : il était allongé dans le petit ravin de Podhoretz, sous la lumière d'une lune pâlissante, qui annonçait l'aube. [...] Soudain la voix de Sarah se fit entendre, et un corps d'une chaleur exquise pesa doucement contre le flanc de Haïm, cependant que des doigts fins écartaient un à un les cailloux :

Comment ça va, dit-elle, tu es bien?

[...]

Je suis bien, je suis bien, répétait-il rêveusement, tandis que de vagues lueurs pénétraient dans la pièce, par la fenêtre où se découpaient un nouveau jour, un ciel nouveau. Le ciel d'Auschwitz inaltéré, inaltérable, comme au premier jour de la Création. (ÉdM, pages finales 248 et 249)

Fig. 7 : Dans L'Homme et la Ville, d'Henri Laborit (1977), ébauches de scènes reprises dans L'Étoile du matin

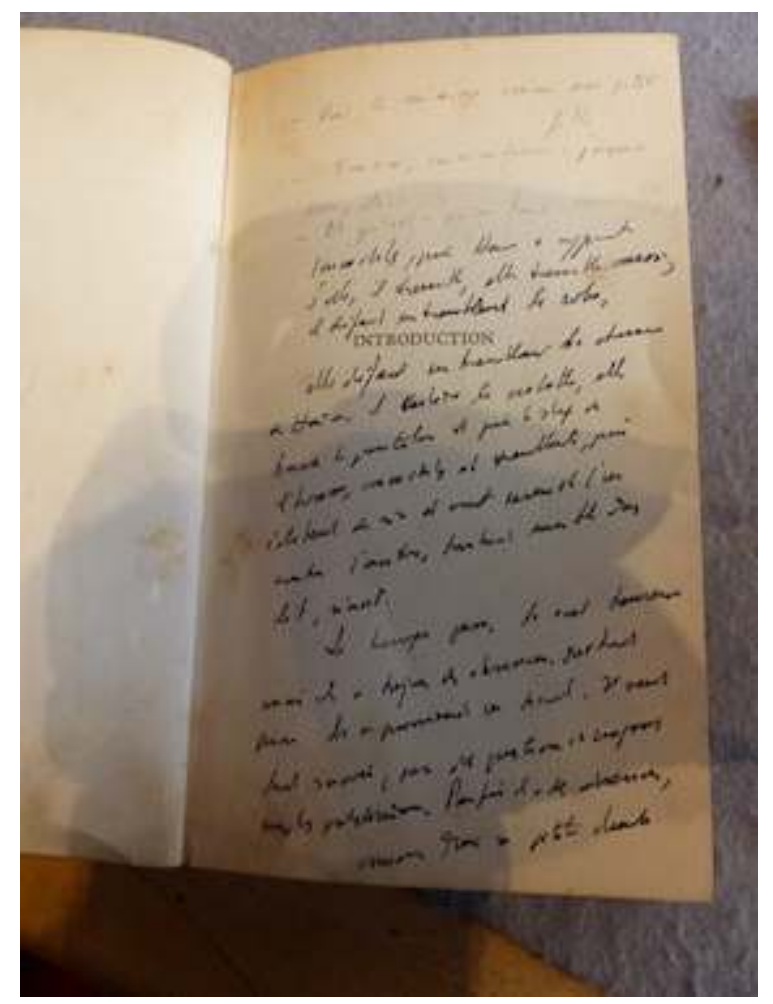

(c) BnF, Fonds Schwarz-Bart

31 Comme on le voit, chez Schwarz-Bart, le lien entre la " source référentielle ${ }^{38}$ » et l'acte endogénétique apparaît distendu voire absent sauf par leur mitoyenneté. Au point que cette "source" se révèle moins comme une référence que comme un catalyseur de l'écriture fictionnelle par association, a-t-on suggéré plus tôt, comparaison ou 
divergence ou, parfois, oserait-on dire, par simple effet mécanique, la présence de mots en suscitant d'autres. L'écriture devenant alors une " apposition ${ }^{39}$ » en marge d'écrits thématiquement similaires ou non, conceptuellement proches ou pas.

Il arrive, bien entendu, que le phénomène intertextuel soit plus classique et que la "source référentielle » se présente comme telle au sens propre. Ainsi un ouvrage de Tzvetan Todorov, Face à l'extrême (Seuil, 1991 et 1994), plus proche des travaux d'André Schwarz-Bart, présente des interactions directes avec l'œuvre en cours dans les années quatre-vingt-dix qui débouchera en partie sur L'Étoile du matin. L'ouvrage suscite en particulier des notes sur les épisodes du ghetto de Varsovie qui occupent le centre du roman de Schwarz-Bart, et certains noms de rues (telle la rue Lezno,) sont repris dans L'Étoile du matin (p. 146). Par exemple, un fait relaté par Marek Edelman ${ }^{40}$ et cité par Todorov à propos d'une jeune fille emmenée du ghetto à Treblinka se retrouve à peine transformé dans L'Étoile du matin :

C'est par exemple l'histoire d'une jeune fille, Pola Lifszyc, qui se déroule au moment où les convois partent pour Treblinka. «En passant chez elle, elle a vu que sa mère n'était pas là. Elle était déjà dans le troupeau poussé ver l'Umschlagplatz et Pola a couru après de la rue Lezno à la rue Stawski : elle a croisé son fiancé qui l'a prise sur son cyclopousse pour aller plus vite et elle y est arrivée. Au dernier moment, elle s'est glissée dans la foule pour pouvoir monter avec sa mère dans le wagon.» (T. Todorov, Face à l'extrême, p. 25. Voir fig. 8)

Et soudain, encadrée par des agents de la police juive, la mère de Rachel apparut dans les rangs, se dirigeant vers les quais de la déportation. "Mamelé, petite maman ", s'écria Rachel avec une sorte de douleur enfantine. Les bras dressés en l'air, elle tourna sur elle-même, égarée. Puis elle se précipita hors de la pièce, dévala l'escalier à toute allure, enfourcha une bicyclette qui trainait dans le couloir et se mit à pédaler afin de rattraper le cortège avant qu'il ait atteint l'endroit où attendait le convoi déjà fumant et sifflant. Quand Haïm arriva à son tour, la place de départ était bouclée, le train s'était déjà mis en marche. (ÉdM, p. 180-181) 
Fig. 8 : Épisode de la fin du ghetto de Varsovie, identifié par A. Schwarz-Bart dans Face à l'extrême de Tzvetan Todorov (1990) et repris dans le roman posthume, L'Étoile du matin (2006)

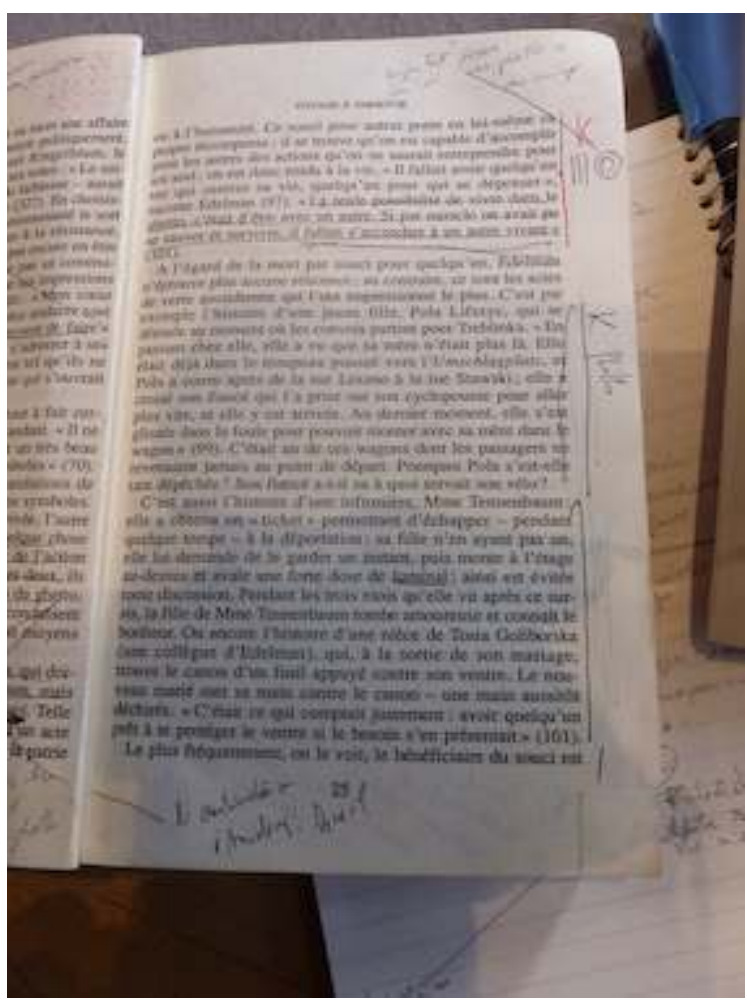

\section{Génétique de la virtualité}

L'Étoile du matin est toutefois très loin d'épuiser le matériel génétique consigné dans la bibliothèque d'André Schwarz-Bart. Si l'on y découvre des ébauches avancées d'épisodes qui seront intégrés dans les pages de la première partie (intitulée "Kaddish »), dictées et/ou dactylographiées à la fin de sa vie par André Schwarz-Bart ${ }^{41}$ et des fragments repris dans la deuxième partie ( Un chant de vie »), le roman publié se révèle comme la pointe d'un iceberg bien plus vaste. Et à mesure qu'on l'explore, il apparaît, avec une évidence de plus en plus criante, que la bibliothèque offre le panorama complexe mais fascinant d'une œuvre qui dépasse L'Étoile du matin dans ses dimensions tant extensive qu'intensive.

Cette œuvre restera à jamais inachevée. Car si la première partie de L'Étoile du matin correspond dans ses grandes lignes au premier volet du projet annoncé en 1985 (un conte ou récit en Pologne), la deuxième partie destinée à décrire l'après-Auschwitz, extrêmement lacunaire, ne semble pas avoir fait l'objet d'une écriture suivie et cohérente. Le déséquilibre en nombre de pages des deux parties dans le texte imprimé l'indique bien: quelque cent quatre-vingts pages pour la première, soixante pour la deuxième alors que la majorité des notes dans les carnets et dans la bibliothèque concerne ce "Chant de vie » censé, aux yeux d'André Schwarz-Bart, redonner espoir aux générations futures après la Catastrophe. Constituer, en somme, le deuxième volet du Dernier des Justes, la renaissance ou la résilience d'un nouveau Juste.

On se doit donc de constater que le kaddish impossible, mais "quand même possible " annoncé dans la lettre à Francine Kaufmann, ne s'est pas concrétisé en écriture. Ce 
qu'André Schwarz-Bart lui-même semble reconnaître et regretter dans une note (voir fig. 9) au sein d'un numéro spécial de la revue Pardès, "Penser Auschwitz ", en marge d'un article d'André Elbaz rappelant la parole de certains Juifs dans les camps de la mort: «dessinez-nous, dessinez-nous, au moins nous resterons vivants sur le papier $^{42} »$ :

[...] certains disent à Haïm ; écris. Mais il n'y arrive pas et délègue la tâche à A.S.

[note complétée ultérieurement, dans une encre plus claire :]

(qui n'est pas arrivé non plus et n'a personne à qui le déléguer : au vent?)

Fig. 9 : Notes successives, en deux encres différentes, d'André Schwarz-Bart sur l'incapacité à écrire dans un texte d'A. Elbaz sur la représentation des camps de la mort

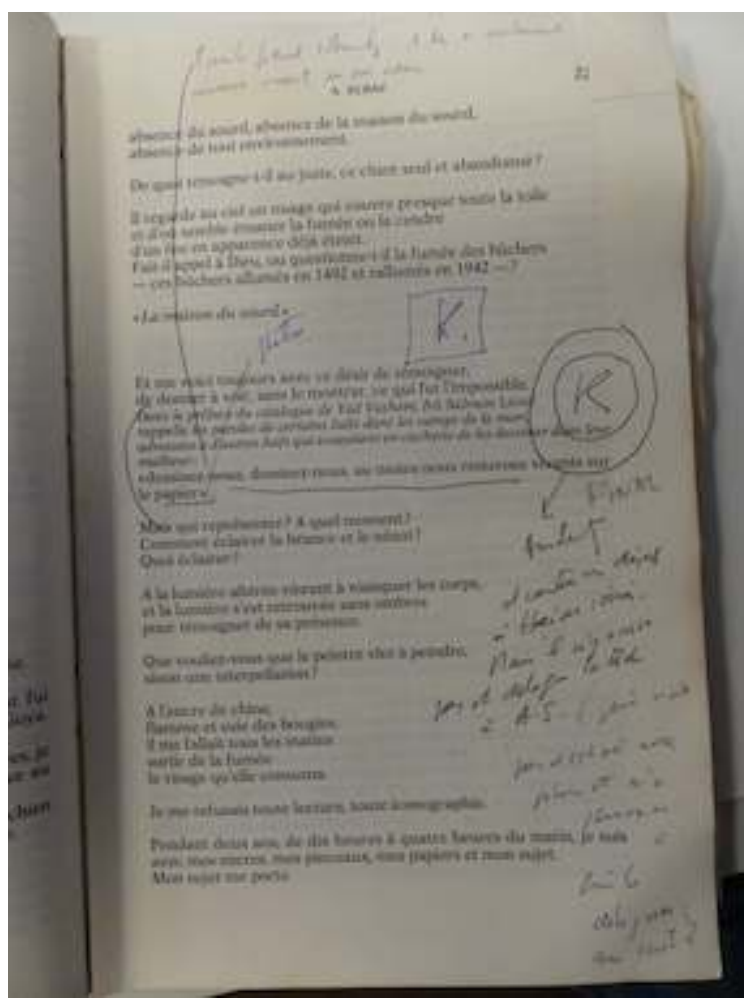

(c) BnF, Fonds Schwarz-Bart œuvre dont la/les virtualité(s) s'expose(nt) comme l'ensemble des pistes qui, à un moment, celui de l'écriture, ont dessiné des aboutissements possibles et même probables.

Ces pistes sont tracées dans une multitude de notes qui parsèment les ouvrages. Elles portent sur la structure de l'œuvre projetée: les plans et plus encore les notes programmatiques sont nombreux. Ainsi, dans une marge d'un numéro de la revue Esprit de juillet 1993 sur « Le poids de la mémoire » :

À Paris, quelqu'un qui a voyagé dans le même convoi raconte la fin de la famille Levy. Moritz revient du camp plein de vie, et c'est après que ça ne s'arrange pas pour lui : désenchantement. Il a des soubresauts : parfois on le croit bien habillé, lumineux, mais c'est passager. Les 2 histoires d'amour et de retour à la vie (la sienne et celle de Moritz Levy) sont donc parallèles.

Les pistes esquissent aussi d'autres dispositifs narratifs qui font intervenir l'ironie notamment à travers le dédoublement du personnage principal en A.S. ou A.S.B. 
(André Schwarz-Bart) et Haïm qui dialoguent comme un homme et son reflet dans un miroir - ou l'autofiction. Les deux, ici, mêlées :

Haïm (Goyave) : J'espère que tu n'as pas l'intention d'écrire q.q. chose sur les Noirs.

A.S. : Pourquoi pas?

Haïm : Tu te condamnerais toi-même (comme ayant osé engager la comparaison).

Déjà que tu n'es pas casher avec ton mariage ; mais si tu essaies d'écrire, etc.

Parfois l'autofiction touche à l'intime, permise par la spontanéité au fil de lectures, cachée entre les livres des autres, plutôt que dans des carnets où l'acte d'écriture est davantage délibéré. Ainsi dans une note où, à travers les initiales, l'on reconnaît l'auteur, son double et son épouse :

K. Guyane. Elle demande à Haïm où il faut enterrer AS.

Haïm : Il faut lui poser la question.

S. : Je l'ai fait. Il a répondu de manière ironique. Mon corps ne me concerne pas: une fois mort, l'homme n'est rien pas plus qu'une grive morte au milieu d'un champ.

Haïm : Il faut l'envoyer à Jérusalem. C'est ce qu'il veut.

S. : J'en étais sûre.

Elle rayonne, et il l'entend fredonner dans la cuisine. Mais lui-même Haïm n'est pas sûr qu'il ne faille pas prendre AS à la lettre ${ }^{43}$.

D'autres personnages que ceux de L'Étoile du matin apparaissent, liés (ainsi Moritz Levy, frère d'Ernie Levy, le dernier des Justes) ou non (Finkelstein ou des anonymes surgis au fil d'épisodes esquissés) à l'œuvre d'André Schwarz-Bart. D'autres acquièrent un rôle majeur, tels le « chroniqueur » ou la « chroniqueuse ».

Des lieux absents du roman posthume prennent une place parfois importante : le Brésil, New York, Goyave en Guadeloupe.

Des thèmes nouveaux sont abordés, comme la dhimmitude, à savoir la situation des Juifs dans le monde arabe. D'autres sont approfondis, tel celui du rescapé des camps de la mort. 
Fig. 10 : Ébauche d'une scène autofictionnelle dans un numéro spécial, « Le poids de la mémoire », dans la revue Esprit

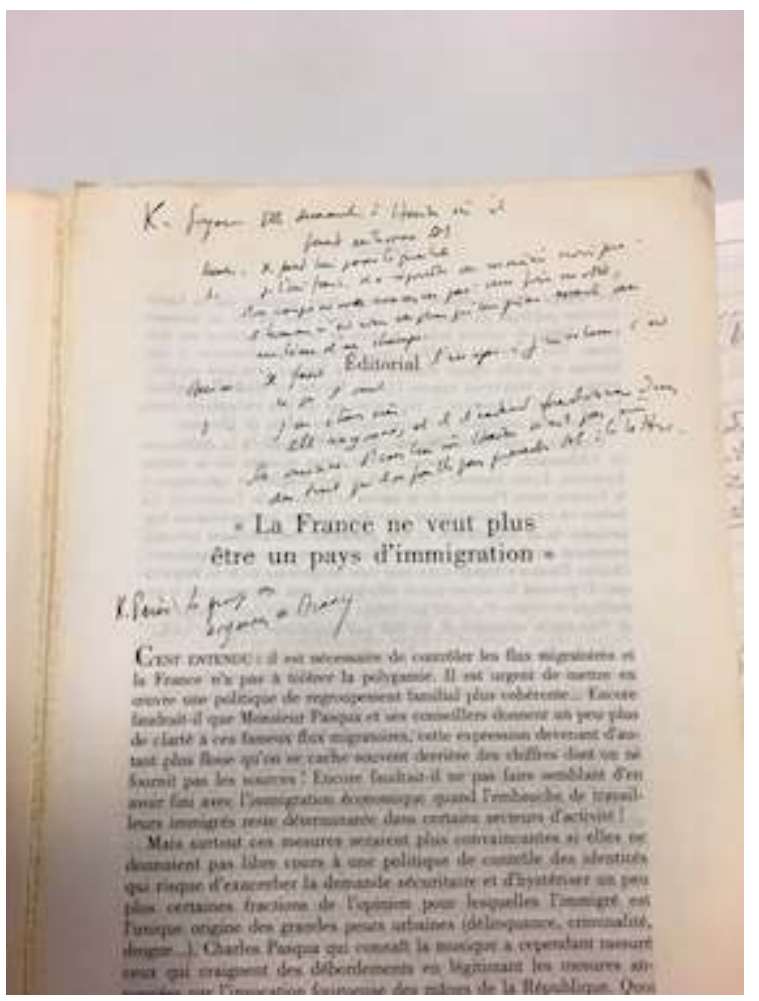

(c) BnF, Fonds Schwarz-Bart

On n'en finirait pas de citer les notes, ébauches, plans, scénarios qui émaillent la bibliothèque, au point que cette profusion sans aboutissement « textuel » nous amène à nous arrêter sur la terminologie à appliquer.

Car si, bien entendu, l'ensemble de ces éléments manuscrits compose, au sens où la génétique le définit ${ }^{44}$, l'avant-texte et devra être agencé (inventorié, classé, daté, déchiffré) de manière critique par les généticiens qui se pencheront sur lui, on peut toutefois s'interroger sur le mot "avant-texte »: certes l'ensemble des "brouillons » (au sens large du terme ${ }^{45}$ ) n'ont de sens et n'ont eu de fonction originelle que de mener à un "texte ». Ils existent donc en tant que tels. Mais ce texte n'ayant jamais ${ }^{46}$ eu d'existence, ou une existence très partielle reflétant peu l'ampleur du projet global, et n'étant pas advenu pour des raisons qui imprègnent l'avant-texte même (incapacité existentielle, impasse autant artistique qu'éthique ${ }^{47}$ ), le mot «avant» qui induit nécessairement un «après » ou un «état définitif » efface la spécificité de cet «avanttexte » (au sens génétique) : son inachèvement structurel qui conduit, paradoxalement, à le poursuivre sans fin. Ou faudrait-il parler d'un "avant " perpétuel, répété, voire bégayé ? Jamais « arrêté » dans l'acception physique du mot, mais aussi juridictionnelle ou politique : jamais l'auteur (et encore moins l'éditeur) ne met fin aux délibérations et ne pose un verdict. Un projet d'œuvre où le telos est ainsi repoussé sans cesse parce que, quasi ontologiquement ${ }^{48}$, il annihilerait le sens de ce qui y a mené, supposant chez l'auteur humain une puissance simili-divine sur l'ordre des choses et la signification des faits.

Peut-on dès lors utiliser le concept de dossier génétique? Oui, si l'on considère que la démarche génétique s'attache à ordonner, dans ce «dossier de genèse ", ce type même 
de documents par nature inachevés tant que l'auteur ne décide pas que son texte est définitif et signe le "bon à tirer » que lui présente l'éditeur. Oui, si l'on considère que le dossier de genèse est le plus souvent composé d'éléments épars, divers, parfois contradictoires ${ }^{49}$ que le généticien séquentialise à partir d'indices en reconstituant un projet qui trouve peu à peu sa cohérence et sa finition au stade textuel et ce, quand il existe, jusqu'au texte imprimé. Oui, si l'on considère que tout dossier de genèse est constitué de versions ou "mondes possibles", éphémères et contrefactuels ${ }^{50}$ et que le processus d'écriture est toujours ou longtemps ouvert jusqu'à ce qu'une «logique " aboutisse à la forme du texte publié $e^{51}$.

Mais si les possibles demeurent dans leur multiplicité et leur virtualité sans que s'opère, structurellement parlant, une logique de la nécessité, si l'hypothétique demeure hypothétique et le virtuel virtuel, ne peut-on penser que l'étude génétique serait ici précisément et uniquement cela : non pas une génétique virtuelle ${ }^{52}$, mais une génétique $\mathrm{du}$ virtuel ou des virtuels, dont on possède les traces (bien réelles) mais sans qu'aucune ne mène à une réalité, à savoir une version finie. Dans le cas de «Kaddish » d'André Schwarz-Bart, il s'agirait dès lors d'une génétique qui intégrerait une étude des probabilités ${ }^{53}$ et présenterait la multiplicité des constructions possibles, à partir des matériaux épars, parfois hétérogènes, trouvés dans les carnets et la bibliothèque ${ }^{54}$. Une pluralité ouverte de textes en puissance que l'auteur n'a pas portés jusqu'à l'acte pour différentes raisons. Toutes celles, philosophiques, citées plus haut. Et, surtout, sans doute, la crainte de fixer dans une structure finie ce qui relevait pour lui de l'infini de la mort et de la tragédie ou encore, davantage, la terreur de faire mourir - en arrêtant leur destin - une deuxième fois les morts dont il voulait parler : «Peut-être était-ce là son véritable objectif: non pas écrire un livre, mais demeurer en contact avec les disparus, leur ménager un espace de vie sur la terre, en son esprit, jour après jour, jusqu'à sa disparition d'ici-bas... » (ÉdM, «Épilogue », p. 251) $)^{55}$. Le fini faisant injure à l'infini, la vie niant la mort, la présence oubliant l'absence.

À charge ensuite au prophète Élie - personnage jamais vraiment mort, aux réapparitions récurrentes dans le folklore juif - d'ordonner l'empilement des notes comme dans le mot hébreu tekou plusieurs fois cité par André Schwarz-Bart, formé de la première lettre des mots tichbi, yetaretz, kouchioth, veabayoth et signifiant « Élie (le Tichbite) résoudra les difficultés ${ }^{56} »$ ! Plus rationnellement, il reste à nous débrouiller avec le matériel laissé, sinon légué par André Schwarz-Bart (car, comme le dit son épouse Simone, il devinait que ses notes consignées dans sa bibliothèque lui survivraient, à l'opposé des manuscrits détruits, comme une bouteille à la mer dans son naufrage), de la même façon qu'il a confié implicitement ${ }^{57}$ le soin à son épouse de retrouver et d'assembler les fragments épars de L'Étoile du matin.

Plus précisément encore, il s'agit désormais de mener une étude approfondie ${ }^{58}$ de la bibliothèque qui établira la ou les cartographies possibles d'une œuvre inaccomplie, déposée dans les livres des autres, on l'a dit, en un territoire conquis. Un espace livresque pénétré par effraction, comme par un errant qui s'installerait dans la propriété d'autrui, ou - pour reprendre une image évoquée plus tôt - un voleur qui, non seulement, rendrait le produit de son vol, mais enrichirait la maison qu'il cambriole, à la fois en y apportant des objets et des richesses, et en l'agrandissant par de nouvelles pièces et une nouvelle structure. 


\section{NOTES}

1. André Schwarz-Bart, L'Étoile du matin, Paris, Seuil, coll. « Cadre rouge », 2009, p. 201.

2. Ancien résistant ayant collaboré à Combat, André Parinaud (1924-2006) co-dirige de 1953 à 1959 la revue littéraire La Parisienne, proche du mouvement des « Hussards » avant de diriger de 1959 à 1967 Arts, où se croisent les mêmes Hussards et les représentants de la Nouvelle Vague cinématographique. André Parinaud est aussi connu pour ses entretiens radiophoniques, notamment avec L.-F. Céline.

3. La réception du Dernier des Justes a fait l'objet d'un mémoire, présenté par Malka Marcovich à l'EHESS sous la direction de Pierre Nora en 1986, La Dernière Rumeur du juste. Le miracle éditorial du Dernier des Justes d'André Schwarz-Bart, Iggybook, 2020. M. Marcovich en a repris les lignes principales dans Le Monde du 5 novembre 2019: «En 1959, le Goncourt d'André Schwarz-Bart faisait resurgir les zones d'ombre de notre histoire collective. » Quant à la genèse du roman, elle a été étudiée par Francine Kaufmann dans sa thèse de doctorat, Le Dernier des Justes d'André Schwarz-Bart: genèse, structure, signification (Paris-Nanterre, 1976), adaptée dans Pour relire Le Dernier des Justes, réflexions sur la Shoah (Paris, Librairie des Méridiens-Klincksieck, 1986).

4. Le parcours d'André et Simone Schwarz-Bart a été raconté par Simone Schwarz-Bart dans un livre de mémoires écrit avec Yann Plougastel : Nous n'avons pas vu passer les jours (Paris, Grasset, 2019).

5. Pour ce qui est de la réception de l'œuvre des Schwarz-Bart dans le public antillais, on lira l'article de Fanny Margras, "Pluies et vents sur Solitude : étude de la réception des œuvres d'André et Simone Schwarz-Bart en Guadeloupe en 1972 ", à paraître en 2021 dans Continents Manuscrits, $\mathrm{n}^{\circ} 16$, en ligne sur openedition.org. L'auteure décèle peu de réserves à l'égard de l'œuvre du couple à la parution du premier volume du cycle dit antillais, du moins dans les Caraïbes elles-mêmes, mais davantage d'indifférence. Le silence éditorial d'André Schwarz-Bart serait le résultat d'un questionnement intime de sa propre légitimité.

6. Nicole Lapierre, Causes communes, Paris, Stock, coll. « Un ordre d'idées », 2011, p. 240.

7. Il épaulera cependant son épouse, Simone Schwarz-Bart, qui a commencé à publier sous son seul nom en 1972 (Pluie et vent sur Télumée Miracle, suivi de Ti Jean L'Horizon, 1979, et de Ton Beau Capitaine, 1987, tous aux Éditions du Seuil). En 1988, Simone Schwarz-Bart publiera, avec la collaboration active d'André, une encyclopédie en six volumes, Hommage à la femme noire (Paris, Éditions Consulaires, 1988-1989).

8. Plusieurs notes de ses carnets évoquent cette crainte, de même qu'un passage fictionnel de L'Étoile du matin: "Il était en deuil de la littérature, en deuil de lui-même. Était-ce la suite de certaines affaires parisiennes? Sans aucun doute, cette meurtrissure survivrait à tout » (ÉdM, op. cit., p. 203).

9. On se référera aussi à l'étude de Francine Kaufmann relative aux raisons du silence éditorial et des destructions de ses textes manuscrits, "André Schwarz-Bart: détruire le livre», dans C. Placial et V. Litvan (dir.), Traces et Ratures de la mémoire juive dans le récit contemporain, Berne, Peter Lang, coll. « Recherches en littérature et spiritualité », 2020.

10. Correspondance conservée à l'IMEC.

11. André Schwarz-Bart, L'Étoile du Matin, op. cit., p. 209.

12. Agencé, complété et publié peu à peu à titre posthume au Seuil par Simone Schwarz-Bart.

13. Même si, selon Francine Kaufmann, André Schwarz-Bart aurait détruit certains des livres de sa bibliothèque, affirmation qu'il est difficile de confirmer à ce stade.

14. Ou des dépôts non retrouvés chez des connaissances (témoignage de Simone Schwarz-Bart).

15. Ces pertes s'ajouteraient à la destruction des documents mentionnée plus haut, pour expliquer leur disparition. 
16. Dont l'inventaire a été établi au début des années 2010 par Elie Duprey, complété par une équipe de bibliothécaires de l'Université des Antilles en 2018.

17. Fait confirmé par Simone Schwarz-Bart dans un entretien avec l'auteur de cet article : « Nous avons acheté parfois trois ou quatre fois le même ouvrage. »

18. On trouve davantage, dans la bibliothèque de Simone Schwarz-Bart, des sources d'Hommage à la femme noire (op. cit.), l'encyclopédie écrite en collaboration avec André Schwarz-Bart. Les ouvrages de cette bibliothèque sont peu annotés par André Schwarz-Bart, relativement à ceux qui l'entouraient dans son espace personnel.

19. Daniel Ferrer, "Un imperceptible trait de gomme de tragacanthe », dans P. D'Iorio et D. Ferrer (dir.), Bibliothèques d'écrivains, Paris, CNRS Éditions, coll. «Textes et Manuscrits », 2001, p. 20.

20. Bulletin de l'Éducation nationale, 17 décembre1959. Interview recueillie par Olga Wormser, citée par Francine Kaufmann, «La non-vocation d'André Schwarz-Bart », Continents manuscrits, $\mathrm{n}^{\circ} 10$, «Devenir écrivain », dir. Céline Gahungu, 2018, mis en ligne le 15 mars 2018 sur openedition.org.

21. Ibid.

22. Cette dénonciation de l'intellectuel bourgeois chez André Schwarz-Bart est confirmée dans des entretiens personnels de l'auteur de cet article avec Simone Schwarz-Bart.

23. Daniel Ferrer, op. cit., p. 20.

24. L'édition originale date de 1946 aux Éditions du Pavois (Paris). L'édition annotée par A. Schwarz-Bart est celle de Hachette, coll. «Pluriel » (poche, 1993, date d'édition manquante dans l'exemplaire d'A. Schwarz-Bart), ce qui ne signifie pas qu'il n'ait pas lu et possédé de précédentes éditions.

25. Daniel Ferrer, op. cit., p. 20.

26. Robert Antelme, L'Espèce humaine, Paris, Gallimard, $1^{\text {re }}$ édition, 1947.

27. Pascal Quignard, Les Ombres errantes, Paris, Grasset, 2002.

28. Alain Finkielkraut et Peter Sloterdijk, Les Battements du monde : Dialogue, Paris, Pauvert, 2003.

29. Viviane Forrester, « Où est l'erreur? ", Le Débat, n 96, 1997, p. 188.

30. David Rousset, op. cit., p. 46.

31. Daniel Ferrer, op. cit., p. 15.

32. Ibid.

33. Pierre-Marc de Biasi, "Qu'est-ce qu'un brouillon? Le cas Flaubert: essai de typologie fonctionnelle des documents de genèse ", dans M. Contat et D. Ferrer (dir.), Pourquoi la critique génétique? Méthodes, théories, Paris, CNRS Éditions, coll. « Textes et manuscrits », 1998, p. 49.

34. Les deux lettres sont des documents personnels de Francine Kaufmann, qui les a cités dans «Le dernier roman d'André Schwarz-Bart, cinquante ans après Le Dernier des Justes, une Étoile du matin en forme de Kaddish », Paris, L'Arche, $\mathrm{n}^{\circ}$ 616, novembre 2009.

35. Simone Schwarz-Bart, «Petite note d'introduction» dans André Schwarz-Bart, L'Étoile du matin, op. cit., p. 14 et 15.

36. Significativement le deuxième prénom de Simone Schwarz-Bart.

37. Henri Laborit, L'Homme et la Ville, Paris, Flammarion, coll. «Champs », 1977, date du tirage manquante.

38. Pierre-Marc de Biasi, « Qu'est-ce qu'un brouillon ?... », art. cit., p. 49.

39. Au sens, non pas grammatical du terme (encore que...), mais physique où l'on appose quelque chose sur autre chose (des scellés, une signature, un visa...).

40. Auteur, notamment, de Mémoires du ghetto de Varsovie: un dirigeant de l'insurrection raconte, Paris, Liana Levi, 1993.

41. Ainsi que le confirme Francine Kaufmann selon ses propres lectures des annotations d'André Schwarz-Bart dans sa bibliothèque (échange électronique du 19 décembre 2019).

42. André Elbaz, «Ne pas montrer l'impossible ", Pardès, nº spécial 9-10, "Penser Auschwitz », Paris, Éditions du Cerf, 1989, p. 227. 
43. Note marginale dans la revue déjà citée Esprit, "Le poids de la mémoire", juillet 1993, éditorial, p. 3, numéro 0113 de l'inventaire personnel de sa bibliothèque par lui-même (fig. 10).

44. Pierre-Marc de Biasi, Génétique des textes, Paris, CNRS Éditions, «Biblis, $10 »$, p. 68-70, et «Qu'est-ce qu'un brouillon ?... », art. cit., p. 39-41.

45. «Un ensemble comprenant à la fois ces documents [les brouillons proprement dits, appartenant à la phase de textualisation] et les manuscrits de travail relatifs à d'autres fonctions (structuration, documentation) mais qui ont été utilisés pour cette textualisation (plans, scénarios, notes documentaires, etc.) ", Pierre-Marc de Biasi, «Qu'est-ce qu'un brouillon ?... ", art. cit., p. 35, voir aussi p. 54-55.

46. Comme dans d'autres cas tel le second volume de Bouvard et Pécuchet de Flaubert. Voir PierreMarc de Biasi, « Édition horizontale, édition verticale. Pour une typologie des éditions génétiques (le domaine français 1980-1995)» dans B. Didier et J. Neefs (dir.), Éditer les manuscrits (archives, complétude, lisibilité), Vincennes, PUV, 1996, p. 180-181, où est décrite la spécificité des éditions de «brouillons ou documents inégalement avancés, inachevés et laissés intégralement à l'état de chantier ».

47. Et non pas parce que l'auteur aurait soit été empêché par le temps, la maladie ou la mort de l'achever, soit l'aurait abandonné par décision ou à la suite d'un blocage définitif.

48. Voire religieusement selon certaines interprétations possibles qu'il conviendrait d'étayer, André Schwarz-Bart s'étant la plupart du temps tenu à distance de la religion, hors de ses dimensions historiques et culturelles.

49. Pierre-Marc de Biasi, « Qu'est-ce qu'un brouillon ?... », art. cit., p. 56.

50. Ibid. Voir aussi Daniel Ferrer, «Le matériel et le virtuel : du paradigme indiciaire à la logique des mondes possibles", dans M. Contat et D. Ferrer (dir.), Pourquoi la critique génétique ?, Paris, CNRS Éditions, coll. « Textes et manuscrits », 1998.

51. Pierre-Marc de Biasi, « Qu'est-ce qu'un brouillon ?... », art. cit., p. 56.

52. Au sens où la proposent les tenants d'une génétique des textes sans manuscrits. Ainsi Patrick Dandrey (dir.), Génétique matérielle, génétique virtuelle. Pour une approche généticienne des textes sans archives, Presses de l'Université de Laval, 2009.

53. En s'appuyant sur la pratique de la critique et de l'édition génétiques quand elles sont confrontées à un projet non abouti et évaluent les propositions des «brouillons " selon des coefficients de certitude, du conjectural au certain en passant par l'hypothétique, et opèrent des simulations par extrapolation (échange de l'auteur de cet article avec Pierre-Marc de Biasi, février 2020).

54. Voir Daniel Ferrer qui évoque la logique modale dans Logiques du brouillon (Seuil, coll. «Poétique», 2011, "Mondes possibles et logiques modales", p. 144 à 147) et parle aussi de «mondes stipulés » (p. 148-150) ou de " prescriptions modalisées» (p. 165). On citera aussi Julien Gracq quand il évoque les «fantômes de livres » dans Lettrines : "Un élément essentiel risque de toujours manquer à la critique littéraire, et particulièrement aux monographies, souvent très volumineuses qu'elle consacre de nos jours à tel ou tel roman célèbre: "La genèse de Madame Bovary", "Les sources des Liaisons dangereuses", etc. Cet élément - sur lequel l'écrivain seul pourrait renseigner - ce sont les fantômes de livres successifs que l'imagination de l'auteur projetait à chaque moment en avant de sa plume [...]. À chaque tournant du livre, un autre livre, possible et même probable, a été rejeté au néant » (Euvres complètes, Paris, Gallimard, coll. «La Pléiade ", vol. III, p. 151). Merci à Jérôme Villeminoz, de la BnF, de m'avoir rappelé ce passage que cite aussi Daniel Ferrer dans Logiques du brouillon (op. cit., p. 166-167). Paul Valéry parlait luimême de "possible-à-chaque instant » : «Ce serait là substituer à l'illusion d'une détermination unique et imitatrice du réel, celle du possible-à-chaque-instant, qui me semble plus véritable » (cité par Bernhild Boie dans la note 151 au texte de Gracq, op. cit.). Si l'écriture de toute œuvre est parsemée de ces possibles avortés, il faut cependant noter qu'André Schwarz-Bart fait de ces avortements ou inaboutissements un état, voire un choix, sinon une œuvre elle-même. Si donc, 
comme le fait remarquer Daniel Ferrer, la critique génétique est exempte de l'infirmité de la critique traditionnelle dénoncée par Julien Gracq puisqu'elle se sert des traces des « fantômes de livres ", on peut même aller plus loin : dans le cas d'André Schwarz-Bart et d'œuvres inachevées similaires, c'est sur ces livres-fantômes que, sans texte les faisant sortir de leur état potentiel, la génétique devrait, nous semble-t-il, se concentrer. Des fantômes enfermés, chez Schwarz-Bart, dans un palais de miroirs. Notons aussi, ici, qu'entre autres significations, le terme "fantôme " désigne une fiche matérialisant l'absence d'un livre dans une bibliothèque.

55. Voir aussi, à ce propos, Francine Kaufmann, «André Schwarz-Bart : détruire le livre », art. cit., à paraître en 2020 .

56. Signification donnée par Arnold Mendel dans La Voie du hassidisme, Paris, Calmann-Levy, 1963, p. 46, note de bas de page. Ouvrage annoté par André Schwarz-Bart.

57. Voir L'Étoile du matin, op. cit., introduction et prologue.

58. À la suite d'un appel à candidature pour une recherche (pos)doctorale sur la bibliothèque d'André Schwarz-Bar lancé en 2020 par l'équipe « Manuscrits francophones » de l'ITEM dirigée par Claire Riffard, un projet postdoctoral de ce type se met actuellement en place.

\section{RÉSUMÉS}

André Schwarz-Bart (1928-2006), auteur du Dernier des Justes, prix Goncourt 1959, a détruit nombre de documents manuscrits de ses œuvres en gestation, dès lors demeurées inachevées ou disparues à sa mort. Dans le «naufrage » de cette œuvre, tel qu'il semble tour à tour le constater et l'organiser, sa bibliothèque apparaît comme un îlot sauvegardé. Durant les vingt dernières années de sa vie, André Schwarz-Bart y mènera un dialogue singulier avec les auteurs l'entourant de leurs pages. Il y consignera aussi plans, esquisses, notes d'intention d'une œuvre, « Kaddish ", qu'il ne mènera jamais à son accomplissement, hormis dans des pages dictées à ses proches. Celles-ci constitueront la plus grande part d'un roman posthume, L'Étoile du matin. L'article tente de dessiner le processus exogénétique entre le texte d'autrui, la note ou esquisse et le roman publié. L'ensemble des notes marginales de sa bibliothèque dépasse cependant de loin ce dernier. L'article s'interroge dès lors sur les notions d' «avant-texte » et de "dossier génétique » dans le cas d'œuvres inabouties et postule une étude des virtualités sans qu'aucune ne débouche sur ce que l'on définit comme « texte».

André Schwarz-Bart (1928-2006), author of Dernier des Justes (Goncourt Prize, 1959), destroyed many of his working manuscripts. As a result, certain works remained unfinished and others vanished with his death. Amid the "wreckage" of his oeuvre, such as he described it and intended it be, his personal library appears as a safe haven. During the last twenty years of his life, André Schwarz-Bart entertained a one-sided dialogue with the books of his library. He also recorded plans, sketches and notes for a work, "Kaddish," which never progressed past sections dictated to relatives. These pages make up the greatest part of his posthumous novel L'Étoile du matin. This article attempts to describe the exogenetic process between the other people's books, his novel's preparatory materials, and the novel itself. However, the notes in the margins of the books in his library are of much greater length than the posthumous novel. The case of unfinished works provides the terrain to question the concepts of the "avant-texte" and the genetic file, and this article theorizes a study of virtualities in which nothing leads to what one may define as a "text." 
André Schwarz-Bart (1928-2006), Autor des Dernier des Justes, Preisträger des Goncourt 1959, vernichtete während deren Entstehung eine Reihe von handschriftlichen Dokumenten seiner Werke, die dann unvollendet blieben oder bei seinem Tod verschwanden. In dem „Schiffbruch“ dieses Werkes, wie er es anscheinend mal entdeckt und mal organisiert hat, scheint seine Bibliothek eine geschützte Insel zu sein. In den letzten zwanzig Jahren seines Lebens wird André Schwarz-Bart einen einzigartigen Dialog mit den Autoren führen, die ihn mit ihren Seiten umgeben. Er wird auch Pläne, Skizzen, Notizen über die Absicht eines Werkes, Kaddish, aufzeichnen, die er niemals zur Vollendung bringen wird, außer in Seiten, die er seinen Nächsten diktierte. Diese werden den größten Teil eines posthumen Romans, L'Étoile du matin, ausmachen. Der Beitrag versucht, den exogenetischen Prozess zwischen dem Text einer anderen Person, der Notiz oder Skizze und dem veröffentlichten Roman nachzuvollziehen. Der Umfang von Randbemerkungen in seiner Bibliothek geht jedoch weit über Letzteres hinaus. Der Artikel fragt daher nach den Begriffen „Vortext“ und „genetische Datei“ im Falle von unfertigen Werken und postuliert eine Untersuchung von Virtualitäten, ohne dass eine davon zu dem führt, was als "Text" definiert wird.

André Schwarz-Bart (1928-2006), autor de Le Dernier des Justes, premio Goncourt 1959, destruyó numerosos documentos manuscritos de sus obras en gestación, que quedaron inconclusas o desaparecidas en el momento de su muerte. En el "naufragio" de esta obra, que parece haber sido constatado y organizado sucesivamente por el escritor, su biblioteca permanece como un islote preservado. Durante los últimos veinte años de su vida, André Schwarz-Bart entablará un diálogo singular con los autores alrededor de sus páginas, en el que quedarán registrados planes, esbozos, notas de intención de una obra, "Kaddish", que no llevará nunca a término, salvo en las páginas dictadas a sus frecuentaciones más cercanas. Éstas constituirán la mayor parte de una novela póstuma, L'Étoile du matin. El presente artículo trata de describir el proceso exogenético entre el texto de otro, la nota o esbozo y la novela publicada. Sin embargo, el conjunto de notas marginales de su biblioteca supera ampliamente este último. En consecuencia, este artículo cuestiona las nociones de "pre-texto" y de "dossier genético" en el caso de obras inacabadas y postula un estudio de sus virtualidades, sin que ninguna de ellas desemboque en lo que se define como "texto".

André Schwarz-Bart (1928-2006), autor de Dernier des Justes, prémio Goncourt 1959, destruiu muitos documentos manuscritos das suas obras em gestação, que desde então permaneceram inacabadas ou desapareceram após a sua morte. No "naufrágio" da sua obra, como ele o parece constatar e organizar, a sua biblioteca aparece como uma ilha salvaguardada. Durante os últimos vinte anos da sua vida, André Schwarz-Bart conduzirá um diálogo singular com os autores das páginas que o cercavam. Ele registará também planos, esboços, notas de intenção de uma obra, "Kaddish", que nunca chegará a concluir, exceto em páginas ditadas a próximos. Estas constituirão a maior parte de um romance póstumo, L'Étoile du matin. $\mathrm{O}$ artigo procura traçar o processo exogenético entre o texto alheio, a nota ou esboço e o romance publicado. $O$ conjunto de notas marginais da sua biblioteca, ultrapassa, no entanto, de longe este último. 0 artigo questiona, portanto, os conceitos de "ante-texto" e "dossier genético" no caso de obras inacabadas e reclama um estudo de virtualidades sem que nenhuma delas conduza ao que se define como "texto".

André Schwarz-Bart (1928-2006), autore de L'ultimo dei giusti, premio Goncourt 1959, ha distrutto molti documenti manoscritti delle sue opere in fase di gestazione, che da allora sono rimaste incomplete o addirittura scomparse dopo la sua morte. Nel "naufragio" di quest'opera, naufragio che egli sembra constatare e addirittura organizzare, la sua biblioteca appare come uno scoglio superstite. Tra queste pagine, durante gli ultimi vent'anni della sua vita, André Schwarz-Bart condurrà un dialogo particolare con gli autori. $\mathrm{E}$ a queste pagine affiderà anche progetti, bozze $\mathrm{e}$ 
note di un'opera - "Kaddish" - che non completerà mai, eccetto alcune pagine dettate ai suoi familiari e che costituiranno la maggior parte di un romanzo postumo, La stella del mattino. L'articolo tenta di descrivere il processo esogenetico tra il testo altrui, la nota o la bozza e il romanzo pubblicato. L'insieme delle note marginali della sua biblioteca è tuttavia molto più vasto del romanzo. L'articolo s'interroga di conseguenza sulle nozioni di "avantesto" e di "dossier genetico" nel caso di opere incompiute, postulando un esame delle virtualità senza che nessuna di queste porti a un qualcosa che possiamo definire "testo".

\section{AUTEUR}

\section{JEAN-PIERRE ORBAN}

JEAN-PIERRE ORBAN est chercheur associé à l'ITEM au sein de l'équipe « Manuscrits francophones ». À ce titre, il est l'auteur d'études, entre autres, sur Sony Labou Tansi (Genesis, $n^{\circ}$ 33) et Yambo Ouologuem («Livre culte, livre maudit : l'histoire du Devoir de violence de Yambo Ouologuem », Continents manuscrits, Hors Série 2018). Depuis 2017, il est responsable du groupe de travail Schwarz-Bart et a impulsé le transfert des archives Simone et André Schwarz-Bart à la BnF. Romancier et biographe, il est notamment l'auteur de Pierre Mertens, Un siècle pour mémoire et de sa version numérique augmentée, Pierre Mertens et le ruban de Möbius (Impressions Nouvelles). 